\title{
IV. SUMMARY OF DISGUSSIONS AND DESIDERATA
}

The meeting was concluded by a summary presented by J. H. Oort and subsequent discussion of the principal desiderata for future work. The following paragraphs include the principal topics of this summary and the desiderata.

\section{Galactic clusters and $O$ associations}

It has become more and more evident how extremely important the study of galactic clusters and $\mathrm{O}$ associations is for the investigation of galactic structure. This is so for three reasons:

(a) For locating spiral structure independent of the velocity. It would be hard to overemphasize this point. We can hope to determine the scale of the Galactic System from them, as well as spiral structure.

(b) Most important of all: they can give information on systematic deviations from circular motion.

(c) They lend themselves excellently for a determination of age, for a determination of change of stellar composition with time and for the derivation of semi-empirical paths of evolution.

The study of clusters may eventually teach us something about differences in distribution and motions of objects formed at various epochs in the development of the Galactic System. What we need is:

(I) To discover more distant clusters.

(2) To find older clusters.

(3) To obtain colour-magnitude diagrams of good accuracy for all clusters. This involves the problem of identifying members.

(4) Radial velocities.

In connexion with the latter item we refer to the report by Weaver on the valuable material on radial velocities of stars in galactic clusters obtained by Trumpler, which is now being prepared for publication.

At the instigation of Drs Heckmann and Haffner the feasibility of obtaining in a number of regions around the sky standard magnitudes and colours to be used for calibrating the photometric data for individual clusters was investigated during the meeting by a small committee. Its proposal, presented by Dr Walker, is given in the Appendix. 


\section{Cepheids}

Kukarkin has stressed again the desirability of searches for more distant $\delta$ Cephei variables. There can be no doubt that this offers one of the most important ways of investigating galactic structure and dynamics. This has been borne out again by the report given by Oosterhoff on the colourexcesses of southern cepheids and the space distribution derived with the aid of these colours.

The amount of work involved in new surveys will be very large, and coordination is therefore important. It is evidently essential that accurate colour-excesses and radial velocities be determined for all $\delta$ Cephei variables that will be found.

\section{Double and multiple stars}

Kulikovsky has proposed that more extensive investigations be made of trapezium-type groups, as well as of wide double and multiple stars in general.

In connexion with the general topic of this conference mention should be made of the fact that a co-ordinating commission for $\mathrm{T}$-associations was formed during the Burakan symposium on Non-stable Stars; members are Haro, Herbig and Kholopov.

\section{Spiral structure}

Since the Groningen conference a great mass of new information on the distribution of neutral hydrogen has become available. The observations concerning the southern parts of the Milky Way are now being reduced. A new development which appears particularly intriguing is the discovery of a spiral arm in the nuclear region which moves away from the centre, at a velocity of $53 \mathrm{~km} / \mathrm{sec}$. Other structures, closer to the centre, show still larger deviations from circular motion.

The report of the Groningen conference stressed the importance of studying possible differences between the distributions of $\mathrm{A}$ stars, $\mathrm{K}$ giants and the neutral hydrogen. Ramberg has reported on his work on A stars and $\mathrm{K}$ giants. Though indicating some relation with the nearby spiral structure of the gas the investigations do not yet show how closely the spiral structure of the gas and stars coincide. The investigations should be extended to larger distances and to more regions before they can give a final answer to this question.

A small working committee was appointed whose task it will be to investigate, how work on the density distribution for different components of the galactic population, in both low and high latitudes, can best be 
promoted. Its members are: A. Blaauw, W. W. Morgan, J. Ramberg, N. G. Roman, and A. Sandage.

\section{Optical work on interstellar lines}

The meeting endorsed a proposal by Kerr and Westerhout, urging the extension of optical work on the velocity distribution of interstellar clouds along the lines of recent work by Münch. Such work is of great significance for the interpretation of $21-\mathrm{cm}$ observations.

\section{$\mathrm{H}$ in regions}

Kerr and Westerhout also urged extension of observations of $\mathrm{H}$ in regions, in view of the interpretation of continuous radio emission at short wavelengths. More detailed radio surveys at wave-lengths shorter than $10 \mathrm{~cm}$ should supplement the available data. In this connexion, also, infra-red surveys are much wanted.

\section{Special regions}

Parenago has made a proposal to study a number of special regions (see p. 6r). Undoubtedly it is of considerable value to concentrate efforts on a few special regions, and those selected are undoubtedly important ones. It was therefore proposed that the conference agrees in principle to the plan and advocates that support be given to it as far as possible. It was stressed at the same time that attention should be concentrated on individual data of very great accuracy rather than on rough data for great numbers of stars. It is still uncertain how much value the determination of proper motions will have in connexion with the study of the space structure of these regions.

\section{Accurate spectrophotometry}

The possibility of accurate spectrophotometric determinations of spectral types, luminosities and possibly a third parameter has been discussed by Chalonge, Strömgren and Miss Roman. The subject had been much discussed at our previous symposium; since then a large amount of data has been collected and the great advances that may be obtained through this type of accurate measurement have been amply demonstrated. Here indeed is an enormously rich mine for observational programmes of the greatest importance for galactic research.

Miss Roman's investigations of high-velocity stars have indicated that the ultra-violet excess is well correlated with average velocity, and may therefore be an important indicator of age for the types of stars she studied. 
O. G. Wilson has recently shown that the width of the emission core of the $K$ line in $G, K$ and $M$ stars is strongly correlated with absolute magnitude, while being independent of spectral type. This might prove to be the most accurate method for determining absolute magnitudes. But high dispersions are required. Co-ordination with other accurate measures of luminosity is important.

\section{Trigonometric parallaxes}

It was proposed that parallax observers would be asked to determine accurate trigonometric parallaxes of about two dozen stars with very weak lines, a list of which will be made available by Miss Roman.

\section{Determination of $\mathrm{K}_{z}$}

This still leaves much to be desired. The best prospects would seem to be offered by accurate objective-prism classifications of $K$ stars down to about I $4^{\circ}$ photographic magnitude in the galactic polar caps, preferably in Kapteyn's Selected Areas where photometric and proper-motion data are already available. It would be desirable to make measurements that would separate giants from sub-giants and dwarfs. Knowledge of radial velocities down to the same magnitude limit would be of the greatest importance.

\section{Radial velocities}

Progress with the objective-prism method, as reported by Fehrenbach, is extremely promising. Similar work will be taken up at Herstmonceux. Co-operation for obtaining a sufficient number of faint standard velocities is desirable.

\section{Nucleus and disk}

The desirability of paying more attention to long-period variables for investigating the older disk population was stressed by Kukarkin and strongly supported by the meeting.

\section{Halo}

The search for RR Lyrae variables in a number of regions in moderate galactic latitudes which was advocated at the Groningen conference has meanwhile been undertaken, and has been reported at this meeting. Complete sets of plates down to a faint magnitude limit have been obtained with the 48-inch Schmidt of Mt Palomar and are now being searched for variables by Dr Plaut at Groningen. An essential desideratum still to be provided is colour excesses for all the variables found.

Morgan has reported on the striking difference in the spectra of various globular clusters. These indicate that there is a class of clusters which are 
relatively rich in heavy elements and which is probably concentrated towards the galactic disk. However, there are likewise considerable differences in composition among clusters having large distances from the galactic plane.

Further investigations of spectra, as well as colours and magnitudes of individual stars in globular clusters, are highly desirable.

\section{Magellanic Clouds}

Thackeray reported on some of the work on the Magellanic Clouds that is now being done or that would seem of importance to be done in the nearest future. This latter comprises a.o. objective-prism surveys to 13.5 photographic magnitude, objective-prism radial velocities to eliminate foreground objects, a search for bright variables, further photometry of known variables and a general search for new ones, in particular also for those of long period. An atlas of charts should be made, covering a larger field than the Harvard charts. 